\title{
Explaining coincidences
}

\author{
Julio De Rizzo ${ }^{1}$
}

Received: 13 May 2021 / Accepted: 8 October 2021 / Published online: 10 November 2021

(c) The Author(s) 2021

\begin{abstract}
A traditional account of coincidences has it that two facts are coincidental whenever they are not related as cause and effect and do not have a common cause. A recent contribution by Lando (Noûs 51(1): 132-151, 2017) showed that this account is mistaken. In this paper, I argue against two alternative accounts of coincidences, one suggested by Lando, and another by Bhogal (Philos Phenomenol Res 100(3): 677-694, 2020), and defend a third one in their place. In short, I propose that how explanatory links relate to non-coincidental facts in explanation is what drives a wedge between coincidences and non-coincidences. This proposal is not susceptible to the worries I raise, and is more general, since it is not restricted to coincidences and non-coincidences involving physical facts.
\end{abstract}

Keywords Coincidence $\cdot$ Explanation · Causation · Grounding

\section{Introduction}

Coincidences are ubiquitous: the CIA agent was at the capital exactly when His Excellency dropped dead (Lewis, 1986); I unwittingly booked myself onto the same cruise as my long lost enemy (Owens, 1992); Bertrand checked his stopped clock exactly at the moment where it got the time right; Ramsey was born on the same calendar day as Schopenhauer; the thirteenth digit (9) of the decimal representation of $\pi$ (3.14 159 $2653589793 \ldots$ ) is the same as the thirteenth digit of the decimal representation of $e$ (2.71 828182845904 5...) (Davis, 1981, cited in Lange, 2010), and so on. Surely, the status of some examples as coincidences is debatable: I pray for nice weather and the day dawns clear and bright; several instants of 'the Dark Side of the Moon' accompany happenings in 'The Wizard of Oz' when both are played at the same time. Apart from deciding upon particular cases, the more interesting question concerns what is at stake, that is, what accounts for some facts being coincidences and others not?

Julio De Rizzo

julioderizzo@gmail.com

1 Department of Philosophy, University of Vienna, Universitätsstraße 7., 1010 Vienna, Austria 
On the face of it, facts being coincidental or not seems to have something to do with explanation. Indeed, a traditional account of coincidences has it that two facts are coincidental whenever none of them causes the other, and they do not share a cause, that is are brought about independently. (The account is readily generalized to cover more than two coincidental facts. See below.) In a recent important contribution, Lando (2017) highlighted a kind of example that casts doubt on the traditional account, namely cases in which coincidental facts have a common cause. Both Lando and Bhogal (2020) offered alternative accounts of coincidences in light of these examples. Their strategies try to motivate a distinctive notion of explanation that tells coincidences and non-coincidences apart. In short, for Lando, a fact might have a cause but not be causally explained; while for Bhogal, explainable coincidences only allow for a kind of explanation that is in a sense worse than the one in play in explanations of non-coincidental facts.

In this paper, I criticize Lando's and Bhogal's accounts of coincidences and defend a third one in their place. My account explores the role and shape of explanatory links, that is what connects an explanation's source (or explanans, typically causes, or grounds) to the explanation's result (explanandum, typically effects, or groundees) in a way that makes the former explanatorily responsible for the latter (Schaffer, 2017a, 2017b; Schnieder, 2010). In short, according to the account I propose, in available explanations of coincidences, links relate differently to the facts explained than in explanations of non-coincidental cases. The resulting account is not susceptible to the criticisms raised against the other proposals, and is more general, since it might cover coincidences not only involving physical facts, in particular those in mathematics. ${ }^{1}$

In the remainder of this section, I will state some preliminaries and assumptions regarding the notions of explanation and coincidence. In $\S 2$, I will state the traditional account and Lando's counterexamples to it. In $\$ 3$ and $\$ 4$, I will argue against Lando's and Bhogal's proposals, respectively. My own account will be presented and defended in $§ 5$. The final section concludes the discussion.

\subsection{Preliminaries}

Let us start by clarifying the notion of explanation. 'Explanation' might be used in at least two senses. First, as the semantic content of certain sentences governed by 'because', 'in virtue of', 'due to', 'makes it the case that', and others. On the other hand, 'explanation' amounts to a particular kind of speech act. As such, an explanation (act) is associated with success conditions, in this case having plausibly to do with it promoting understanding, being somewhat 'illuminating', eliminating surprise, and so on (Achinstein, 1983; Bromberger, 1992; Lewis, 1986; Schnieder, 2015). In light of this distinction, one should keep in mind that explanations (content) might be true irrespectively of whether explanations (acts) drawing on them are successful. Thus, an act that aims at explaining why it is true that snow is white while relying on the explanation (content) expressed by e.g. 'It is true that snow is white because snow is white' would hardly be successful in most contexts, in spite of the 'because' claim

\footnotetext{
1 Lange (2010). Indeed, the account might be seen to have relevant points of contact with Lange's account of mathematical coincidences. I will discuss this in due course.
} 
correctly indicating a reason why the truth ascription holds. If not otherwise stated, the content sense of 'explanation' is meant in what follows. I will concentrate on 'because'-sentences as the means of expressing explanations. ${ }^{2}$

'Because'- sentences might be used to express explanations why, as in 'The window shattered because the rock was thrown with force F in trajectory $\mathrm{T}$ at it', or ' $\{$ Socrates $\}$ exists because Socrates exists'. (I will consider only explanations why - as opposed to e.g. explanations how - in what follows.) In these, the sentence on the right of 'because' expresses a cause and a ground, respectively, of what the sentence on the left states. In general, a sentence of the form ' $p$ because q', which is governed by 'because' in its explanatory use, is true if and only if some explanatory relation (e.g. causation, grounding, maybe others and combinations resulting from chaining the ${ }^{3}$ ) holds between what is expressed by 'p' and 'q' (Schnieder, 2010, 2015). For the sake of simplicity, I will speak of the semantic values of declarative sentences as facts, referred to by a term resulting from 'the fact that' concatenated with a true declarative sentence. ${ }^{4}$ The resort to facts indicates that explanations are factive in the sense that sentences flanking 'because' in a true sentence governed by it are themselves true. Following standard practice, I will assume that explanations are asymmetric and transitive, that is: if $\mathrm{p}$ because $\mathrm{q}$, then it is not the case that $\mathrm{q}$ because $\mathrm{p}$; and if $\mathrm{p}$ because $\mathrm{q}$ and $\mathrm{q}$ because $r$, then $p$ because $r^{5}$

In addition to the explanandum and the explanans, a further role is played in explanations by what one may call explanatory links ('links' for short). Accordingly, explanations have a tripartite structure: First, the explanans, or source; second, the explanandum, or result; and third, the link that connects source and result in an explanatory way (Schnieder, 2010; DeRossett, 2013, Schaffer, 2017a, 2017b; Kappes forthcoming). Thus what connects the fact that the rock was thrown with force F and trajectory $\mathrm{T}$ at the window and the fact that it shattered is the fact that under certain conditions objects with a certain mass and momentum break solid glass structure when colliding with it; while the fact that Socrates exists and the fact that $\{$ Socrates $\}$ exists

\footnotetext{
2 This contrasts with an evidential use of 'because', as in 'The Smiths are home because their light is on', and 'It is cold outside because the thermometer shows 5 degrees Celsius'. In these, the sentence on the right of 'because' does not provide a reason why the sentence on the left holds, but perhaps only a reason why one came to believe that it does. See Morreall (1979) for discussion.

3 I do not want to exclude that further explanatory relations, alongside causation and grounding, be accepted as holding between explanandum and explanans of explanations why. Moreover, 'more specific' relations might play this role in place of grounding, for instance the relation between determinates and determinables, set membership, part-whole, and so on, provided they all conform to the formal features ascribed in the text. (Similarly with causation.) The locus classicus of a conception of explanations as 'tracking' explanatory relations - sometimes called 'dependence' or 'determination' relations - is Kim (1994). It is allowed that the explanatory relations at stake be only partial (partial causation, partial grounding, and so on).

4 For a prominent defense of facts as relata of causation, see Mellor (1995).

5 Given the truth-conditions for 'because' sentences just stated, this implies that explanatory relations and disjunctions of them are asymmetric and transitive as well. Besides, plausibly, since constraints of relevance apply to the obtaining of explanatory relations in general, this carries over to 'because' sentences. In particular, they come out non-monotonic, in the sense that addition of sentences to the right-hand side of true 'because' statements might not preserve truth. To be sure, while these are standard assumptions, they have been the subject of much discussion I cannot engage with here. They are here stated for the sake of completeness, and will not play a prominent role in what follows.
} 
are linked by the fact that sets' existences are grounded in the existence of their members (I shall discuss more examples below). These facts play the explanatory role of links, which are distinct from that of source and result in an explanation. ${ }^{6}$

Following a common take on this, I assume that links are lawlike explanatory patterns among facts, however these patterns are ultimately to be understood (for instance as mere regularities in a Humean fashion, as relations between universals, or the like). For the sake of space and generality, I shall not try to give necessary and sufficient conditions for a fact to function as an explanatory link. This touches on intricate issues concerning the concept of law, which is a debate on its own. At a minimum, for present purposes links should satisfy two main constraints: i) they should be general, that is apply to all objects of a domain specified in the link; and ii) be expressible solely by means of predicates that are natural or sparse in the Lewisian sense (Lewis, 1983), and purely qualitative, that is, with no ineliminable mention of specific individuals (under some conceptions of naturalness, this second condition is superfluous). Though these constraints leave open whether sentences expressing links mention explanatory relations (causation, grounding, or others) explicitly, I shall assume that links determine what facts function as explanatory sources and results in every case (more on links below). ${ }^{7}$

I turn now to the notion of coincidence. Firstly, there seems to be a sense of 'coincidence' tying it to a feeling of surprise, or unexpected outcome. Though I do not deny that this restricted sense might be prevalent in everyday talk - dictionary entries provide evidence of this-, I shall not rely on it: for all that follows, there are many 'boring' coincidences, such as that I washed my hands today at the same time the Queen of England walked her dogs. From a methodological standpoint, such a lenient sense is preferable. For otherwise the question we started with gets blurred by which psychological factors might make a fact surprising, and this plausibly brings with it a relativization to subjective expectations, background knowledge, and so on. ${ }^{8}$

Relatedly, I will not restrict coincidences to those involving some kind of 'match' between facts, as it is the case for instance with Schopenhauer and Ramsey being both born on the same calendar day, or two people in a supermarket aisle wearing the same pair of 1980's red Nike Air Ship high-tops: if Schopenhauer were born 17 calendar days earlier than Ramsey; or if one of the two people in the supermarket were wearing brown All Stars and the other flipflops, these facts would also be coincidences. ${ }^{9}$ In the same vein, I will assume coincidental facts, that is facts 'making up' a coincidence,

\footnotetext{
6 How exactly links relate to the corresponding 'because' sentence is a substantive question I will not enter into. A plausible suggestion is that a link grounds the fact that (result) obtains because (source) obtains. In this sense, links are higher-order reasons why their corresponding explanations obtain (Schnieder 2010; Skow 2016). Nothing in what follows hinges on a particular choice on this.

7 It might be that we must make room for yet a fourth explanatory role in play in explanations to account, for instance, for background conditions. I will leave these out of discussion for simplicity.

8 This contrasts with e.g. Hart and Honoré (1959). Owens (1992), Lange (2010) agree with the view adopted here. Though Lando 2017 and Bhogal 2020 express sympathy towards the restricted sense, their accounts do not essentially rely on it. No point in my criticism relies on this difference in our frameworks, and the account I shall offer might be easily adapted to apply to the more restricted sense.

9 Both Lando (2017) and Bhogal (2020) express sympathy to a restriction of coincidences to such 'matching' facts, and indeed consider only examples in line with this restriction. I will come back to this shortly.
} 
need not be simultaneous ${ }^{10}$ : that my cat stepped on the piano and played the first three notes of 'When you wish upon a star', or that I woke up today 22 min after my neighbor did are also coincidences. Though I will discuss only coincidences 'consisting' of more than one fact, I do not rule out that simple facts might turn out coincidental (however odd this sounds given the original meaning of 'co-incidental'). As a tentative example, consider a hungry donkey that stands in front of two exactly similar bales of hay. It might turn out a coincidence that it chose a particular one over the other. (Maybe a variable taking a particular value at random would also provide an analogous case.) If such cases are accepted as coincidences, the accounts below should be read as restricted to coincidences consisting of more than one fact.

Finally, I will assume there is a single concept of a coincidence applicable in general, for instance both to natural phenomena and mathematical facts, which our initial question aims at. Again, in light of initial evidence that we apply the same notions in both spheres, at least as a working assumption towards an account of coincidences, this seems recommendable. ${ }^{11}$

\section{The traditional account}

That some facts are coincidental seems to entail that no explanatory relation holds between them. In particular, among two coincidental facts, one cannot be a cause of another. (Following the accounts I will engage with, in this and the following two sections, I will concentrate on cases of causation, leaving other explanatory relations aside.) If, for instance, my enemy knew that I booked the cruise and did the same in order to encounter me there, the fact that we boarded the same cruise is not a coincidence, since my having booked the cruise caused them to do so. If Ramsey's parents were fans of Schopenhauer and planned his birthday based on this, the fact that both philosophers were both born on February 22 would not be a coincidence, since the fact that Schopenhauer was born that day would be a cause of their decision leading to Ramsey's birth.

Moreover, two facts need not be themselves related by causation, but the existence of a common cause seems already sufficient to exclude their co-obtaining from being a coincidence. For instance, whenever the leading woman of a given travelling theatre group gets ill, the same happens to the leading man of the same group. As it happens, they both eat together at the same restaurants and whenever they got ill, this was because they had shared tainted food. Intuitively, the latter is sufficient for rendering the former fact not a coincidence.

Wrapping these points together, we arrive at the claim that coincidental facts must apparently be causally independent, that is, not only must causation not hold between

\footnotetext{
10 To be more precise: either the facts themselves need not obtain at the same time (if facts obtain relatively to times); or the time parameter in sentences stating them need not refer to the same time (if facts obtain 'eternally'). Nothing hinges on a choice between these conceptions.

11 Notice that, since mathematical facts hold of necessity, this rules out, for instance, simple accounts of coincidences in terms of the low probability of their joint obtaining which one might initially feel inclined to hold. Both Lando (2017) and Bhogal (2020) explicitly leave out non-physical coincidences. My criticism of their accounts does not hinge on this. See footnote 13.
} 
them, but they must not share a causal source. Turning this into a sufficient condition, these considerations prompt the following view of coincidences ${ }^{12}$ :

The Traditional Account ('TA' henceforth):

It is a coincidence that $\mathrm{p} \wedge \mathrm{q} \wedge \ldots \leftrightarrow$ df none of the facts that $\mathrm{p}$, that $\mathrm{q}, \ldots$ partially causes one of the others; and some facts among them do not have a common (partial) cause*.

Given that facts have complex causal histories, 'common (partial) cause' should here be understood as excluding remote causes. Otherwise, if for instance the Big Bang is a common cause of any (physical) facts, the definition would be empty. This is not to deny that it is true that the Big Bang, or the fact that the Big Bang occurred, is a common causal source, but rather just to stress that, in TA, there is an implicit restriction to non-remote, somewhat salient common (partial) causes (flagged by '*'), however this notion might be more precisely understood (Owens, 1992).

TA finds considerable support in the literature, and it indeed does well with respect to a wide range of cases (Horwich, 1982; Sober, 1984; Owens, 1992) builds his account of explanation on it). However, in spite of its initial plausibility, TA should be rejected, due to a class of counterexamples highlighted by Lando (2017).

Consider the case of a kid playing in a courtyard with a ball. She throws the ball high, it bounces off of the balcony of one apartment, then of the balcony of another. In each there is a grand piano. As it happens, the ball hits the same note, the high A, on both keyboards. The fact that the same note is struck on both pianos is apparently a coincidence. Or consider the following case. There are a hundred urns lined up in a row, each with a hundred balls numbered 1-100. They are connected by a switch, the flipping of which triggers the following process: a ball is drawn from the first urn, and its number, $n$, is recorded; then a ball is drawn in the $n$-th urn. Both selections are unbiased. You flip the switch and, as it happens, the ball 4 is drawn from the first urn, then a ball 4 is drawn from the fourth one. That two balls with the same number were drawn is a coincidence. (I will refer to this example as 'the urn case' in what follows.) In both of these examples, there is a common cause of two coincidental facts, which speaks against the right-hand side of the definition being a necessary condition for coincidences.

Now as some remarks above indicate, I believe one has rather good independent reasons to deny that the fact that the same note is struck on both pianos is a coincidence as the first case is described. For plausibly the fact that the ball hit the high A on the first keyboard is a cause of the fact that the ball hit the high A on the second: it plays along in changing the trajectory so as for the latter fact to obtain as it did. Just as the fact that Ramsey and Schopenhauer were born at the same calendar day would not be a coincidence, had Ramsey's parents paid a tribute to the German philosopher; and

\footnotetext{
12 Where 'p', 'q' stand for true atomic declarative sentences. The other accounts stated in the text follow this regimentation. As stated, it is allowed that only some of the facts 'in' a coincidence do not share a cause. One could easily tweak the account to obtain a more stringent notion, according to which every pair of facts 'in' a coincidence should fulfill this condition. Nothing substantive hinges on this point.
} 
the fact that I and my enemy met would not be a coincidence if my boarding on the cruise were a cause of his, the fact that the same note is struck in both pianos cannot be a coincidence if the hitting of one note causes the hitting of the other. (Lando might have a reply to this I will consider in the next section. Notice that the urn case is not objectionable on the same grounds.) ${ }^{13}$

It would be better, then, to modify the first example. Suppose the kid plays in the courtyard with a delicate wooden airplane this time. She throws the airplane high with such a force that, on its way up, it splits into two parts. The first part hits an A on the piano in the one balcony, the second hits an A on the piano in the other balcony. The fact that the same note is hit on both pianos is indeed a coincidence, and yet the facts involved share a common cause (the kid's throw), contra TA. I will refer to this modified example as 'the piano case', and focus on it in the discussion that follows. Although Lando and Bhogal do not consider this example explicitly, clearly their treatments of the original case should be applicable to this case as well.

Given that similar examples might be construed across the board, I will leave aside a dismissal of these as genuine counterexamples, and refer the reader to Lando's lengthy and convincing defense (see esp. Sects. 4 and 5 of her paper; they apply to the piano case as I've construed it). I will consider two related reactions to the failure of TA, one by Lando (2017) herself and a more recent one by Bhogal (2020). Though I will argue that they should ultimately be rejected, they both have a grain of truth that points to the account I shall propose in due course.

Before proceeding, let me stress an important caveat. I will assume throughout that Lando and Bhogal aim at a definition, that is an account of what a coincidence is. This is admittedly a strong reading of their enterprises: they might be read more modestly as simply offering a different take on how coincidences relate to explanation, without attempting at a novel definition of the notion in these terms. However, their remarks surely hint at accounts of coincidences of interest in their own right. At any rate, my criticism of their views would also apply under a weaker reading of what they are up to.

\section{Lando's account}

Lando writes at the very beginning of her paper that 'it is a truism that coincidences cannot be explained' (2017: p. 1). In effect, this truism hints at her final account of the matter: while coincidental facts might share a common cause, the fact that they both obtain cannot be causally explained.

\footnotetext{
13 I should highlight that I do not deny that some of us have the pre-theoretical intuition that the fact that an A was struck on both pianos, as Lando construes the case, is a coincidence. Our concept of coincidence is arguably, however, far from unified. Under one understanding, a fact being a coincidence seems simply to turn on its low probability of obtaining (plausibly, this notion of coincidence admits of gradation, just as probability does), and this might accommodate the intuition mentioned. My focus here is on a concept of coincidence relating it to explanation, and as announced one that applies to causal and non-causal, including mathematical, cases. (Of course, how this notion relates to probability is a perfectly legitimate, yet still a separate question.) Under this concept, we seem to have good systematic grounds to deny that, when a fact causes another, the fact that they both obtained is a coincidence, just as we do in the analogous examples cited in the main text. Thanks to an anonymous referee for discussion on this.
} 
To be more precise, Lando takes a coincidence to be what she labels a 'relational fact': in the piano case, that the same note is struck on both pianos is a coincidence. On her account, the existence of a common cause of the facts that the high A is struck on the first piano, and the fact that the high $\mathrm{A}$ is struck on the second, is not sufficient in explaining the relational fact itself. She writes:

The fact in need of explaining is that the same note is struck. Even if we could show, separately, why the note struck on the first piano was an A and why the note struck on the second piano was an A, it seems to me we would not yet have explained why it is that the same note was struck on the two pianos. Why not? This last fact is a relational fact; the former are not. To explain the relational fact, it is not enough to show separately why each of the relata had to be what it was. We want a reason for the relationship itself, the fact that the notes matched. (13)

I will mention further details of Lando's account as we proceed. For now, let me highlight that two distinctions are here center stage: between relational and non-relational facts, on the one hand; and between causation and causal explanation, on the other. Both distinctions are in play in the core claim her view rests on, namely, that relational facts might be caused, but not causally explained.

Let us then begin by asking: what exactly is a relational fact? In the case at hand, she states the relational fact as the fact that the same note was struck on both pianos. A natural semi-formalization of this would be ' $\exists \mathrm{x} \exists \mathrm{y}$ ( $\mathrm{x}, \mathrm{y}$ are notes $\wedge \mathrm{x}$ was struck on piano $1 \wedge \mathrm{x}$ was struck on piano $2 \wedge \mathrm{x}=\mathrm{y}$ )'. (Of course, the relation between the note on the first and the second piano is in some sense redundant, since it is simply identity. But if one allows for other more substantive relations between coincidental facts, this semi-formalization might give a better clue to the common structure of relational facts.) If this is on the right track, the fact stated seems to be, in a sense, explainable: since existential generalizations obtain because of its true instances, and conjunctions because of its conjuncts (Schnieder 2011), we have it that the same note was struck on both pianos because (A is a note, A was struck on piano 1, A was struck on piano $2, \mathrm{~A}=\mathrm{A}) .{ }^{14}$ Granted, this is hardly an illuminating explanation. But one might go further. The fact that the kid threw the airplane with force $\mathrm{F}$ and with a certain trajectory $\mathrm{T}$ causes the fact that an A was struck on the first piano and causes the fact that an A was struck on the second piano. By a general form of transitivity, we have it now that the same note was struck on both pianos because ( $\mathrm{A}$ is a note, $\mathrm{A}=\mathrm{A}$, the fact that the kid threw the airplane with force $\mathrm{F}$ and a certain trajectory $\mathrm{T}) .{ }^{15}$ Again, this seems a true explanation, and in some sense a better candidate than the former. If Lando's account is correct, one should then either deny the semi-formalization above;

\footnotetext{
${ }^{14}$ I assume that one might state a plurality of facts - facts 'taken together' - as explanans via a list of sentences.

15 As an anonymous referee remarks, the assumption of transitivity combining causal and non-causal explanations is contentious. Especially in the context of the circularity objection against Humean accounts of laws of nature, the validity of this assumption has been subject of intense debate. See e.g. (Miller, 2015; Hicks \& van Elswyk, 2015) for purported counterexamples; Marshall (2015) and Lange (2018) for discussion. A proper discussion of the issues raised must be left for another opportunity. Importantly, notice that the point in the text might be framed without this assumption, by relying instead 'directly' on the last explanation. Indeed, this strategy has the advantage that it dispenses with a specific regimentation of relational facts. Thanks to the anonymous referee for the references.
} 
reject one of the steps in this derivation; or else say that the notion of explanation that drives a wedge between non-coincidental and coincidental facts is stronger than the one exemplified by this (perhaps even, in Lando's terminology, I shouldn't be speaking of 'explanation' at all here).

The first reply would deem relational facts mysterious, and an alternative reading would run the risk of being ad hoc, designed to make the account work. The second reply, in turn, is arguably not attractive. For the derivation of the explanation just sketched turns on largely accepted features of 'because' in its explanatory use, which, absent more compelling reasons to the contrary, one should care to preserve.

The third reply, then, seems the most promising. She indeed indicates that her response turns on the distinction between 'having a cause' and 'being (causally) explained':

In general, we should be quite wary of inferring facts about what can or cannot be explained from facts about what caused what. For to explain is not simply to cite a cause. I argued above that in Pianos [the piano case], the throw of the ball caused each of the A notes to be struck. By separating causation from causal explanation, however, we can still maintain that there is no explanation for the fact that the same note was struck. (12; my square brackets)

(Notice that Lando could use the same distinction to resist my argument against the original piano case above. Accordingly, the fact that the ball hit the A on the first piano is a cause, but does not causally explain, the fact that the ball hit the A on the second piano. My criticism of her account, to be stated shortly, applies to this move as well.)

The following account of coincidences is motivated by these remarks:

\section{Coincidences (I):}

It is a coincidence that $\mathrm{p} \wedge \mathrm{q} \wedge \ldots \leftrightarrow_{\mathrm{df}}$ none of the facts that $\mathrm{p}$, that $\mathrm{q}, \ldots$ partially causally explains one of the others; and some facts among them do not have a common causal (partial) explanans*.

As motivated by Lando, Coincidences (I) relies heavily on the distinction between being caused and being causally explained. As she points out, a distinction along these lines has precedent in the literature. She mentions Davidson, who prominently argued that causation is an extensional relation between coarse-grained events, while explanation is non-extensional, and holds between more finely individuated entities, maybe events under a description, or facts under a suitable conception of these (Davidson, 1967).

Notice that, its acceptability notwithstanding, a distinction along these lines does not by itself suffice to justify Lando's assessment of the piano case (as she is surely aware). For, if one adopts a Davidsonian framework, one might indeed hold that some causal claims do not directly give rise to explanatory claims: for instance, the cause of Caesar's death caused Caesar's death, but that the cause of Caesar's death occurred does not explain why he died. However, even if one accepts this, it is extremely plausible 
that when events are causally related, there must be some description of them under which an explanation holds (or analogously between two corresponding facts). Thus the cause of Caesar's death was his stabbing; and that Caesar was stabbed explains why Caesar died. Hence, armed with the distinction, still a further step is needed to get Lando's account off the ground: namely, with respect to the piano case, that there are no descriptions of the throw of the airplane and the landing of its parts (or that there are no facts 'corresponding' to these events) such that they explain the fact that an A was struck on both pianos. Even if restricted to the cases at hand, this is a strong claim.

Lando should thus insist that in coincidences, all candidate explanations in terms of common causes are somehow spurious, or at any rate do not succeed in explaining why the relational fact holds. But, with respect to the piano case, it is at least questionable that this is a promising route to take (Bhogal, 2020: pp. 5-7 raises similar concerns). Consider, for instance, the facts specifying the trajectory, force, mass and aerodynamics of the airplane toy, positions and resistance of the keys, and so on. Given sufficient details and appropriate links, an explanation citing these facts enables one to predict exactly that the two parts of the airplane thrown by the kid will hit an A on the first piano and hit an A on the second. It also plausibly shows even that both coincidental facts are probabilistically related, since, again given sufficient amount of detail provided in the description of the case, the throwing of the airplane makes it likely that the parts land where they did, and the same note is struck on both pianos (cf. Bhogal, 2020: pp. 6-7). And this seems to explain why the same note was struck, indeed in a successful way, in line with established scientific practice: if this is not a 'good' explanation of why the relational fact holds, one might wonder what a successful explanation in other cases, including non-coincidental ones, could be. ${ }^{16}$ In short, the explanation via the common cause does not seem to leave much room for discontent for the distinction gestured at by Lando to apply to. Once one allows that such common causes are available, the prospects of an account of coincidences in general that denies that they are causally explained look dim. ${ }^{17}$

On Lando's behalf, it should be highlighted that there are surely many ways of spelling out the distinction between causation and causal explanation, let alone different accounts of these notions themselves, and one could not possibly ensure that none of them delivers the result Lando's account relies on. Be that as it may, a weaker point seems still to hold, namely, that insofar as her account turns on specific ways of conceiving of such a distinction at the expense of others, available rival accounts of coincidences that do not are, other things being equal, preferable.

\footnotetext{
16 Lando's account might seem better suited to deal with the urn case, since intuitively it is more acceptable that, even if the flipping of the switch is a cause of a ball 4 being drawn in both urns, by itself it does not seem to explain why the fact that a ball 4 was drawn in both urns obtained. This might be motivated by the fact that the drawings are by assumption randomized, so one cannot determine what the outcome of the drawing would be. Notice, however, that one might alternatively make room for this intuition by saying that an explanation in terms of the flipping is simply a partial one, though of course no less explanatory because of that. Since my criticism of Lando need not rely on this example, I shall not adjudicate between these alternatives. I thank an anonymous referee for discussion of this point.

17 Thanks to an anonymous referee for discussion of my objection against Lando's account.
} 


\section{Bhogal's account}

Bhogal (2020) criticizes Lando's account for closely related reasons, and offers a different take on the case.

With respect to the original piano case presented by Lando, Bhogal acknowledges that the fact that the kid threw the ball with force $\mathrm{F}$ and trajectory $\mathrm{T}$ (and further details) explains the relational fact (what corresponds to what he calls 'the matching proposition') that the same note was struck on both pianos. ${ }^{18}$ I will suppose that he would say the same with respect to the piano case as I've construed it. In contrast to Lando's proposal, his strategy consists in spotting a difference between the kinds of explanation available in non-coincidental and coincidental cases. Namely, he claims that in coincidental cases, the only explanation one might give of the corresponding relational fact is via an explanans that is too specific for it: in the piano case, for instance, an explanation in terms of the specific trajectories, forces and masses of the objects involved (what he labels 'the trajectory explanation' with respect to the original piano case). This difference in specificity makes it allegedly a bad explanation, since the relational fact holds in more physically possible worlds than the purported explanans. In the same vein, the same explanans succeeds in explaining the particular facts that an A was struck on the first piano and that an A was struck on the second, since such a difference of specificity is not available (at least not to the same degree) (Bhogal, 2020: p. 9). He writes:

Intuitively, the trajectory explanation is a bad explanation of the matching proposition in Pianos [the piano case] because it is too tied to the specific way in which the same note was played in the actual world. Explaining why the same note was played on both pianos by appealing to the exact details of the throw ignores all the non-actual ways in which the same note could have been played. (13, my square brackets)

He goes on to spell out in more precise terms an account of explanations based on a specificity criterion, that is, that candidate explanantia should be weighed against each other according to how they modally match their explananda. (As he notes, an account of explanation along these lines finds precedent in the literature.) I take it that, if we were to turn these remarks into a definition of coincidences, then, Coincidences (I) would be broadly in line with Bhogal's account, with the important difference that the notion of causal explanation employed therein incorporates this more stringent constraint. $^{19}$

Without going into the details of the account, let us apply the general idea to the piano case. As I understand it, it takes off from the observation that the fact that the

\footnotetext{
18 Bhogal speaks of coincidences as events and of propositions describing them as the relata of explanation. Though this might conceal more than just a terminological choice, nothing in what I will have to say turns on a difference on this score, and my talk of facts might readily be translated into Bhogal's framework.

19 As an anonymous referee notes, given that Bhogal acknowledges different grades of specificity involved in assessments of explanations in general, it is far from clear that Coincidences (I), even if read as suggested in the main text, does full justice to his account. For the sake of simplicity, and since my criticism aims more at the underlying idea than at particular renderings of his account, I set these issues aside. See the previous footnote.
} 
same note was struck on both pianos might obtain in different ways than it did in actuality (in Bhogal's terms, that the proposition that the same note was struck on both pianos might be true in different ways than in actuality). That is, that same fact might have obtained if the trajectory would have been different in some respects, and in virtue of this for instance a high $\mathrm{D}$, instead of a high A, were struck by each of the parts of the airplane on both pianos. Since the actual explanans in terms of the trajectory, force, and further details of the throw does not carry over to these alternative physically possible scenarios, it allegedly provides for a worse explanation than one that did. As suggested by his remarks, according to Bhogal's proposal, the availability of such explanations of the 'right modal grain' is what tells apart coincidences from non-coincidences as such.

As will become clearer shortly, I do think that the case has something to do with specificity, but not in the way suggested by Bhogal. The first point to be raised against his proposal is a comparatively minor one. Namely, that his account turns heavily on the contention that coincidences must involve a 'matching' between facts, or - to speak in his own terms - that a coincidence must be describable truly by a matching proposition, such as that the same note was struck on both pianos, or that the same number was drawn from both urns. As already mentioned above, this seems hardly justifiable: would it be any less coincidental if the first note were an A while the second a B, that is, would it not be a coincidence that the first part of the airplane struck an $A$ on the first piano and the second part $a B$ on the second one? I take it that, intuitively, one is inclined to regard this and analogous cases as coincidences (at least once one classifies the matching cases as such). Since in these no matching fact (or proposition) of the right sort seems available, Bhogal's account cannot do justice to this intuition. Furthermore, by claiming otherwise one runs the risk of letting a seemingly contingent feature of our conceptual framework (e.g. our musical system with respect to the piano case) - for instance that we classify this and that frequencies under the same notion (A), or conversely that we do not have one notion under which A and B would be the same -, play a determining role as to what should count as a coincidence. This speaks against an objective conception of coincidences that is arguably desirable in contexts linking it to an objective notion of explanation.

Let me turn to the second, in my view weightier, point. According to Bhogal, what makes facts coincidental is that the explanation available for them does not obey some constraints on specificity: in particular, the explanation of a coincidence is bad when compared to non-coincidental cases because the only available explanantia hold in less physically possible worlds than the coincidence itself. Quite independently of the previous point, it might be challenged that such a difference in specificity is as determining of the status of a coincidence as the account suggests. To illustrate this, suppose that there are two photons $a$ and $b$ that exist in the same medium, say in vacuum, in every physically possible world (if this supposition strikes you as too costly, read this simply as a thought experiment to drive a point home). They both traveled, on a particular observed occasion, at the same speed. One might wonder whether this fact is a coincidence. Now one might say that they both traveled at the same speed simply because $a$ travelled at $c$, and $b$ traveled at $c$ (one might supply further details regarding their trajectories, particles in the vicinity and so on). Alternatively, one might point to the fact that photons in free space travel at the speed of light $c$. 
As it happens, both of these candidate explanantia score equally well in terms of their 'modal grain' with respect to that of the explanandum (one might suppose that they are the only explanantia available for the sake of the example). But which of them brings out the status of the first fact as non-coincidental? I take it that the latter is a better candidate, or at any rate that the former fails at this. ${ }^{20}$ But if this is right, then modal strength does not seem involved in what tells explanations of coincidences and non-coincidences apart: even if the modal strength of an explanans in terms of the particular facts matches that of the explanandum itself, it still cannot do the job on its own, as it were, of rendering a fact a non-coincidence. ${ }^{21}$ This example hints at the account I will propose, to which we now turn.

\section{A novel account}

In common with Lando's and Bhogal's proposals, I take it that what is at stake turns on the acceptance of a distinctive sort of explanation which is not applicable to coincidences. That is, there is indeed some feature of explanations of non-coincidental facts that tells them apart from coincidences. While Lando proposed that this feature is simply that non-coincidental facts are explainable, while coincidences are not (even if coincidental facts share a cause), Bhogal proposed that the responsible feature of explanations of coincidences is that their explanantia are too specific for their explananda in a certain sense. In contrast, the proposal I will defend concentrates on how explanatory links relate to explananda in explanations of coincidental and non-coincidental facts.

As a prelude, it is instructive to consider the following mathematical case. Consider the sum of the first $n$ natural numbers: $1+2+\ldots+(n-1)+n$.

When $\mathrm{n}$ is even, we can pair the first and last numbers in the sequence, the second and second-to-last, and so forth. The members of each pair sum to $n+1$. No number is left unpaired, since $n$ is even. The number of pairs is $n / 2$ (which is an integer, since $n$ is even). Hence, the sum is $(n+1) n / 2$.

When $\mathrm{n}$ is odd, we can pair the numbers as before, except that the middle number in the sequence is left unpaired. Again, the members of each pair sum to $n+1$. But now there are $(n-1) / 2$ pairs, since the middle number $(n+1) / 2$ is unpaired. The total sum is then the sum of the paired numbers plus the middle number: $(n+1)(n-1) / 2+(n+1) / 2$. This expression simplifies to $(n+1) n / 2$-remarkably, the same as the formula we just derived for even n. (Lange, 2010: p. 308)

\footnotetext{
20 Even if the fact observed does not obtain in every physically possible world, then the second explanans matches it worse than the first, since it is modally stronger (i.e. obtains in more physically possible worlds) than the explanandum.

21 Notice that his account does not by itself provide a reply to my argument against the original piano case by Lando above. For since presumably the fact that the ball struck an A on the first piano and the fact that the ball struck an A on the second are of the same modal grain, one cannot say that the first causes but does not causally explain in Bhogal's sense. Since he does not consider this argument, and might want to reject it on other grounds, I will not place much weight on this. I thank an anonymous referee for a very helpful discussion of the objections against Bhogal's account.
} 
Thus when $n$ is even, the sum is $(n+1) n / 2$; when $n$ is odd, the sum is also $(n+1) n / 2$. One might wonder whether this is a coincidence. But the following proof shows that it is not:

$$
\begin{aligned}
& S=1+2+\ldots+(n-1)+n \\
& S=n+(n-1)+\ldots+2+1
\end{aligned}
$$

If we pair the first terms, the second terms, and so forth in each sum, then each pair adds to $(n+1)$, and there are $n$ pairs. $S o 2 S=n(n+1)$, and hence $S=n(n+1) / 2$. (Lange, 2010: p. 310)

In contrast to the former, this proof speaks against it being a coincidence that, for even $n$, the sum of the first $n$ natural numbers is $(n+1) n / 2$, and the same holds for odd $n$.

As a further instructive example, take the following curiosity (Sorensen (manuscript) cited in Lange, 2010, pp. 308-9). As it happens, all the numbers produced by pressing three digits on a row, column or main diagonal of a calculator keyboard and backwards (123321, 321123, 456654, 654456, 789987, 987789, 147741, 741147, $258852,852258,369963,963369,159951,951159,357753$, and 753357) are divisible by 37 . One might wonder whether this is a coincidence. As it turns out, it is not, as shown by Nummela (1987: p.147; cited in Lange, 2010):

This is no coincidence. For let $\mathrm{a}, \mathrm{a}+\mathrm{d}, \mathrm{a}+2 \mathrm{~d}$ be any three integers in arithmetic progression. Then

$$
\begin{aligned}
& a \cdot 10^{5}+(a+d) \cdot 10^{4}+(a+2 d) \cdot 10^{3}+(a+2 d) \cdot 10^{2}+(a+d) \cdot 10+a \cdot 1 \\
& =a\left(10^{5}+10^{4}+10^{3}+10^{2}+10+1\right)+d\left(10^{4}+2 \cdot 10^{3}+2 \cdot 10^{2}+10\right) \\
& =1111111 a+12210 d=1221(91 a+10 d) .
\end{aligned}
$$

So not only is the number divisible by 37 , but by $1221(=3 \times 11 \times 37)$.

Once again, the availability of a proof of this sort supposedly shows that the fact is no coincidence, just as the lack of an analogous one concerning, say, the fact that the thirteenth digit (9) of the decimal representation of $\pi$ (3.14 $1592653589793 \ldots$ ) is the same as the thirteenth digit of the decimal representation of $e(2.71828182845$ $9045 \ldots)$, indicates that the latter is a coincidence. But what drives a wedge between the first and the latter two proofs?

Following Lange (2010), I take it that the proofs just given all show not only that the corresponding facts hold, but also why they hold. Given that the first proof above does not dissolve the coincidence, as it were, the answer to our question does not lie in the latter arguments showing why the corresponding facts hold.

Rather, the feature of explanations responsible for the latter two proofs establishing that the facts in question are not coincidental lies, first, i) in them subsuming each of the facts composing the coincidence under a natural pattern: the fact that for even $n$ the sum of the first $n$ numbers is $n(n+1) / 2$ and the fact that for odd $n$ the sum of the first $\mathrm{n}$ numbers is $\mathrm{n}(\mathrm{n}+1) / 2$ are somehow explained by the fact that for $\mathrm{n}$ a natural 
number, the sum of the first $\mathrm{n}$ numbers is $\mathrm{n}(\mathrm{n}+1) / 2$. Similarly, each of the facts that one of the numbers in the second case is divisible by 37 is in a sense explained by the general fact that for $\mathrm{a}, \mathrm{a}+\mathrm{d}, \mathrm{a}+2 \mathrm{~d}$ any three integers in arithmetic progression, $\mathrm{a} \cdot 10^{5}+(\mathrm{a}+\mathrm{d}) 0.10^{4}+(\mathrm{a}+2 \mathrm{~d}) 0 \cdot 10^{3}+(\mathrm{a}+2 \mathrm{~d}) \cdot 10^{2}+(\mathrm{a}+\mathrm{d}) 0.10+\mathrm{a} \cdot 1=(37$ $\times 33)(91 a+10 d)$. Such a pattern is not to be found with respect to the facts that the thirteenth digit (9) of the decimal representation of $\pi(3.141592653589793 \ldots)$ is the same as the thirteenth digit of the decimal representation of $e(2.71828182845$ $9045 \ldots$.. . And, second ii) in these principles not only acting as links in explanations of each of the facts singularly, but of the obtaining of all these facts jointly, that is, their conjunction. ${ }^{22}$

Both conditions are also applicable to non-mathematical cases. Thus take the piano case. The two facts that the one part of the airplane struck a high A on the first piano and that the other part struck a high $\mathrm{A}$ on the second piano do not conform to a natural pattern: no general fact concerning a natural type they together conform to is available. To be sure, they each do admit of an explanation in terms of the trajectory of the airplane, its aerodynamics, force exerted by the kid when throwing it, and one might proceed to explain why the same note was struck on both pianos, and the corresponding conjunction, on the basis of this. But no general claim unifying these facts and acting as a link in an explanation of why they both obtained, that is why the first part struck a high A on the first piano and the other struck a high A on the second piano, is actually telling of an underlying natural pattern they both, jointly, conform to. For instance, the laws of motion acting as links treat both facts separately: after the split, the objects run separate ways, each being subject to independent intervening factors and forces, even if they be taken as parts of a single dynamic system. Though as discussed above they do enable an explanation of the fact that the same note was struck on both pianos in terms of the common cause, the links themselves do not predict that the outputs will relate in any specific, non-trivial way.

Contrast this with the following case. A bullet is shot with a trajectory parallel to the ground. Simultaneously, the cartridge case is dropped from exactly the same height. Is it a coincidence that the bullet and the case land at the same time? No. For the law of gravitation ensures that both objects will be attracted to the ground with the same acceleration, thus that they land at the same time (we abstract away air resistance). Notice that the same would be true, even if the trajectories were not to share a common cause (if two objects were dropped by different people at the same time and from the same height, say). In these cases, the link applies to the facts jointly (in part due to the fact that they fall from the same height, and more importantly that the same body, the Earth, attracts the objects), that is, it gives the conjunctive fact at once as an explanatory result, and subsumes the objects involved, insofar as they are physical bodies, under a natural pattern.

Let me clarify. As stated at the beginning, the role of an explanatory link is that of connecting an explanatory source, or explanans, to an explanatory result, or explanandum. These links might be considered, in effect, as general facts consisting of lawlike

\footnotetext{
22 A natural alternative to framing the explanation of facts jointly is to allow for facts collectively to play the role of explanandum, thus also for 'because' statements to apply to pluralities of sentences on both sides. See Dasgupta (2014) for a defense of this in the context of grounding. Since this calls for a revision on the grammar of 'because' adopted, I stick to the formulation in the text.
} 
patterns between facts of certain types, for instance that true disjunctions are grounded in their true disjuncts, that sets' existences are grounded in the existence of their members, that water expands under $4{ }^{\circ} \mathrm{C}$, that light travels at $c$, that gases expand upon decrease of pressure, that velocities of light rays in air and water cause them to refract, and so on. Crucially, in a sense, one might say that links in general explain their corresponding explananda as well: That the lower part of the oar in a rowboat immersed in water seems to be bent upwards is explained by the fact that the velocities of light rays in air and water cause them to refract; That the beer bottle in the freezer cracked is explained by the fact that water is caused to expand by a decrease in temperature under $4 \mathrm{C},\{$ Socrates \}'s existence is explained by the fact that sets' existences are grounded in the existence of their members, and so on (see Kappes forthcoming). ${ }^{23}$

Now explanatory links and the conjunctive facts they explain might relate in at least two different ways. Take, for instance, the fact that 123321 is divisible by $37 \wedge 321123$ is divisible by $37 \wedge \ldots$ (where '...' covers the other fourteen numbers specified previously). The reason why this fact holds appeals to the conjuncts themselves: 123321 is divisible by $37 \wedge 321123$ is divisible by $37 \wedge \ldots$ because 123321 is divisible by 37 , 321123 is divisible by $37, \ldots$. Now this explanation might be 'backed' by distinct sorts of link. The link might be, for instance, simply that conjunctions are grounded in their conjuncts; or that a number is divisible by another whenever the result of dividing them is a natural number different from 0. Arguably, the first is too general, while the second does not target the conjunction in question specifically enough. Thus either way, these links do not single out the facts involved as conforming to a distinctive pattern. They contrast sharply with the link that for $\mathrm{a}, \mathrm{a}+\mathrm{d}, \mathrm{a}+2 \mathrm{~d}$ any three integers in arithmetic progression, $\mathrm{a} \cdot 10^{5}+(\mathrm{a}+\mathrm{d}) 0 \cdot 10^{4}+(\mathrm{a}+2 \mathrm{~d}) 0 \cdot 10^{3}+(\mathrm{a}+2 \mathrm{~d}) \cdot 10^{2}+$ $(a+d) 0.10+a .1=(37 \times 33)(91 a+10 d)$ : the latter shows that the link between the conjuncts and the conjunction is a regularity that targets the obtaining of the facts involved jointly, that is to say subsumes all of them, together, under a natural pattern. According to my proposal, the availability of an explanation with this kind of link is what draws the line between non-coincidences and coincidences.

As a final illustration, consider an example of a non-coincidence by Kitcher (2001) (cited in Bhogal, 2020). In the eighteenth century, John Arbuthnot noticed that more males had been born than females in London in each of the previous 82 years. This might be stated as a conjunction: the number of births of males $>$ the number of births of females in year $1623 \wedge$ the number of births of males $>$ the number of births of females in year $1624 \wedge \ldots$ (where '...' covers the remaining 80 years after that). Once again, this obtains because the conjuncts obtain (one might supply further details in each case, the exact numbers, and so on). Now one available link might again simply be that conjunctions are grounded in their conjuncts. A more informative one would for instance cite general facts concerning physical, biological and chemical details of the individual copulations leading to each of the male and female births in each year. Yet another kind of link would be that as a matter of evolution, sex ratios tend to an equilibrium of 1:1. (Since the death rate in humans of males is greater than in females, the birth of males will be greater to achieve this equilibrium.) Again, of these, only

\footnotetext{
23 Importantly, given the treatment of 'because' sentences here adopted, this does not imply that the corresponding 'because' sentences involving links are true. See Skow (2016), Kappes and Schnieder (2016) and Roski (2018) for discussion.
} 
the latter sort of link shows that the conjuncts together obey a natural pattern. The availability of this sort of link, relating as it does to these conjuncts as explanatory result, shows them not to be coincidental.

Let us say, then, that in non-coincidences, there is an explanation whose link unifies the facts involved. The account of coincidences I am proposing then reads:

Coincidences (II):

It is a coincidence that $\mathrm{p} \wedge \mathrm{q} \wedge \ldots \leftrightarrow_{\mathrm{df}}$ none of the facts that $\mathrm{p}$, that $\mathrm{q}, \ldots$ partially explains one of the others; and it is not the case that there is a $\operatorname{link} L$ such that $L$ unifies the fact that $p$ and the fact that $\mathrm{q}$ and...

The notion of unification is reminiscent of the account of explanation prominently defended by Friedman (1974) and Kitcher (1981, 1989). In simplified terms, this account has it that explanations are arguments that instantiate argument patterns in the most unifying theory with respect to a range of phenomena to be explained: that is, from which one is able to deduce them with the least number and greatest simplicity of argument patterns. Of course, this notion of unification differs from the one employed in Coincidences (II). For one, the former relates whole theories to a set of phenomena, while the latter relates one fact (the link) and other facts. Still, they certainly share a common idea in the background, namely, that the subsumption of facts under common patterns of a distinctive sort (be they stated in the form of argument patterns, or in the form of general facts, as in our case) plays a determining role in the context of explanations (be it to determine what explanation is, or simply as to what should turn out coincidental, in the present case).

Unification in the present sense obeys some constraints on specificity. On the one hand, unifying links cannot be too general, so as to leave out the subject matter of the conjuncts involved. This excludes, for instance, general principles concerning logical types of facts from being able to unify in the sense intended (for instance, that conjunctions are grounded in their conjuncts) which would be applicable in every case. In other words, lawlike patterns must be selective, or non-trivial, in order to render facts in a conjunction non-coincidental. (Cf. Kitcher, 1981 on the role of nonlogical vocabulary in the condition of stringency on argument patterns; pp. 517-8; pp. 527-528.) On the other hand, it is required of links that they not be too specific, so as to not be applicable to a significant range of similar cases. This is built-in in the very notion of a link we started with, since it excludes, paradigmatically, gerrymandered kinds such as 'being a number of births of male humans in year 1623', or 'is a sample of hexed salt'.

To be sure, I doubt that one can define unification in the sense intended. Importantly, any definition will necessarily turn heavily upon a previous choice of what facts might function as explanatory links in the first place. Relative to such a choice, let us say that a fact playing the role of a link, and satisfying the latter constraints, unifies other facts 
stated in a conjunction only if i) the former is a link in an explanation of the latter; and ii) the objects mentioned in the conjunction all fall under the natural type mentioned in the link as pertaining to the explanatory result. These two necessary conditions give us the means to evaluate Coincidences (II) with respect to the examples of coincidences above, while they help shed light on what renders other examples noncoincidental.

Consider again the piano case. The fact that the kid threw the airplane with force $\mathrm{F}$ and trajectory $\mathrm{T}$, with suitable statements on the material and aerodynamics of the object, explains why the airplane split into two, and each of the parts landed where they did, say at positions P1 and P2 (where the high A keys of the pianos were located). The explanatory results are, then, the facts that part A landed at P1 and that part B landed at P2. However, plausibly 'landing at position P1' and 'landing at position $P 2$ ' (even with fuller spatial specifications) are not mentioned in any single link as part of the explanatory result. Even if the laws of motion, for instance, might predict that each of the parts will land in such and such positions given the initial conditions, they permit that output positions of each vary independently according to the latter, and thus leave plenty of room for variation as to the place where each of the objects will land. As a result, the example fails condition ii), since no lawlike pattern relates facts falling under a natural type to any specific output concerning the positions of the two objects together, with the exception of the special cases where they are subject to the same forces and further conditions. The latter is what happens in the bullet and cartridge case example: taking off from the same height, and being attracted to the Earth, gravitation commands that the time both objects will land is the same (again, we abstract away air resistance). Analogously, the fact that for $a, a+d, a+2 d$ any three integers in arithmetic progression, $\mathrm{a} \cdot 10^{5}+(\mathrm{a}+\mathrm{d}) 0.10^{4}+(\mathrm{a}+2 \mathrm{~d}) 0.10^{3}+$ $(a+2 d) \cdot 10^{2}+(a+d) 0.10+a \cdot 1=(37 \times 33)(91 a+10 d)$ ensures that the fourteen numbers specified as above all fall under the type being divisible by 37 , and the fact that sex ratios tend to an equilibrium of 1:1 'dictates' (here to be understood with a strength applicable to predictions in biology) that, given the difference in death rate, more males tend to be born than females in any of the years registered by Arbuthnot.

Notice that, plausibly, one should allow for heterogeneous lawlike patterns to play the role of links. Suppose I touch a hot pan, my hand twitches away, and I experience pain. ${ }^{24}$ Suppose further that, in general, facts concerning the phenomenology of pain are grounded in certain neurophysiological facts. Intuitively, that my hand twitches away and I experience pain is not a coincidence. But how would the link in this case look like? There are several options to pursue here, but a natural conjecture is that the link involved in an explanation of an explanandum $E$ by an explanans E' will somehow incorporate the links involved in every step of a given path from E' to E. To illustrate with a simple example, consider the following claim: '(the window shattered $\vee$ snow is white) because the rock was thrown at the window'. Plausibly, this is arrived at by two steps: first the explanation expressed by '(the window shattered $\checkmark$ snow is white) because the window shattered', then that expressed by 'the window shattered because the rock was thrown at it'. The former claim, then, has as a link

$\overline{24}$ I thank Yannic Kappes for discussion of this example. 
a heterogeneous pattern, somehow involving both the fact that true disjunctions are grounded in their true disjuncts, plus the fact that objects colliding with solid glass with a certain mass and momentum break glass structure under certain conditions (or something along these lines). ${ }^{25}$ In the hot pan case, then, the link responsible for it not being a coincidence that my hand twitches away and I experience pain involves the grounding of pain by a particular neurophysiological state plus further links involved in the explanatory chain from the twitching to the pain, even if no explicit mention of this is made in the statement of the fact itself. (In the previously discussed examples of non-coincidences, it seems indeed natural to allow for the fact that conjunctions are grounded in their conjuncts to take part, together with the other ones, in the links of the corresponding explanations.) Albeit more complex, these links might still unify the facts pointed as explanatory result in the sense here intended.

In contrast to the previous accounts, Coincidences (II) holds for coincidences in general, that is, it applies to coincidences involving physical as well as non-physical facts. The account has important points in contact with Lange's (2010) proposed account of mathematical coincidences. He writes:

The components of a non-coincidence have a unified explanation of a kind that the components of a coincidence lack. Not all proofs (or perhaps even all explanations) of the components of a mathematical noncoincidence succeed in unifying them. But the fact that those components have a unified explanation makes their truth non-coincidental. (320)

Of course, the similarity lies not just in our use of the same word ('unification'): more importantly, Lange stresses that coincidences might have common explainers (i.e. explanantia stated by premises in explanatory proofs), but the coincidental facts are not unified by one common explanatory proof. ${ }^{26}$ As he points out, Kitcher (1989) also lays emphasis on the shape of arguments of non-coincidences in mathematics, as opposed to their simply being explained (i.e. having explanantia as premises in explanatory proofs). A full comparison between these accounts and the present one would require more space than I can concede here. Leaving aside some of the details, the present proposal shares with them this insight: for, inasmuch as links state patterns connecting explanans to explanandum, they plausibly determine the shape of proofs in which they are used (the premises and conclusion must satisfy what links 'point to' as explanans and explanandum, respectively), and thus are arguably at least partially responsible for the availability of a common explanatory proof of non-coincidental facts.

It is worth mentioning that the present account sits well with the cognitive attitudes we commonly bear to coincidences. In particular, if the availability of lawlike patterns is linked to a particular kind of understanding why (Friedman, 1974), the account

\footnotetext{
25 The 'involving' is deliberately vague here, but it is natural to consider that the new link is simply a conjunction of the previous ones; or perhaps we might simply allow that many facts, as a plurality, play the role of link in an explanation, just as we allow for in the case of explanans. Nothing hinges on a choice on this point. The acceptance of heterogenous links has a bearing on the discussion on the validity of a generalized form of transitivity of explanation mentioned in footnote 15. I must leave a proper discussion of this for another opportunity.

26 An explanatory proof is an argument that shows not only that its conclusion holds, but why it does.
} 
renders it natural the intuitive claim that, in a sense, we do not understand why a coincidence holds (even if some explanations of it, not in terms of unifying links, are available). Moreover, as it happens most coincidences are not expected, or have an element of surprise in some sense. Again, that the coincidental facts do not conform to a known lawlike regularity seems a promising starting point to make sense of this.

Finally, Coincidences (II) gives the right verdicts with respect to non-coincidental common cause cases: that a decrease in atmospheric pressure causes storms and the weight of mercury in barometers to rise acts as a link in the explanation of the fact that the barometer level fell and the storm came in terms of the decrease in pressure; that people eating the same tainted food makes them sick acts as a link in an explanation of the fact that both the leading woman and the leading man of the theatre group got sick; and so on. These are natural patterns, plausibly statable in more precise terms, but which nonetheless unify the facts explained in a way that establishes that they are not coincidental.

\section{Conclusion}

At present, the most promising accounts of coincidences are but attempts to make sense of the intuitive claim that coincidences cannot be explained. When coincidences are restricted to physical facts, that is facts that might be causes and effects, a natural view has it that coincidental facts are causally independent. In a recent important contribution, Lando (2017) showed that this view is mistaken.

Recently, the debate moved on to target the sense of 'explanation' under which the intuitive claim might still be held. In this paper, I argued that two proposals pursuing this aim fail. In sum, Lando's proposal rests on a distinction between being caused and being causally explained whose application to coincidences remains doubtful. In turn, Bhogal's (2020) proposal draws on a difference in modal specificity between explanans and coincidence to pinpoint the difference between explanations of coincidental and non-coincidental facts. I argued that his proposal turns on too restricted a view of coincidences, and thus fails as a general account of the matter. In addition, I called into question that a difference in modal specificity is indeed what tells the explanations of coincidences and non-coincidences apart.

In their place, I proposed that we should look into explanatory links for a satisfactory account of what a coincidence is. Accordingly, coincidences cannot be explained in the sense that every explanation of coincidental facts fail to show that they can be jointly subsumed under a natural pattern, which would correspond to a suitable link. This account not only evades the objections raised, but it also delivers the right results upon examination of examples discussed in the literature, and allows us to do justice to a general and attractive notion of coincidence, applicable in principle to both the physical and the non-physical realms. ${ }^{27}$

\section{Funding Open access funding provided by University of Vienna.}

\footnotetext{
27 I thank Martin Glazier, Yannic Kappes and Stefan Roski and further members of the Forschungskolloquium in Hamburg for discussion of an earlier draft of this paper; and two referees of this journal for helpful discussion.
} 
Open Access This article is licensed under a Creative Commons Attribution 4.0 International License, which permits use, sharing, adaptation, distribution and reproduction in any medium or format, as long as you give appropriate credit to the original author(s) and the source, provide a link to the Creative Commons licence, and indicate if changes were made. The images or other third party material in this article are included in the article's Creative Commons licence, unless indicated otherwise in a credit line to the material. If material is not included in the article's Creative Commons licence and your intended use is not permitted by statutory regulation or exceeds the permitted use, you will need to obtain permission directly from the copyright holder. To view a copy of this licence, visit http://creativecommons.org/licenses/ by $/ 4.0 \%$.

\section{References}

Achinstein, P. (1983). The nature of explanation. Oxford University Press.

Bhogal, H. (2020). Coincidences and the grain of explanation. Philosophy and Phenomenological Research, 100(3), 677-694.

Bromberger, S. (1992). On What We Know and What We Don'T Know: Explanation, Theory, Linguistics, and How Questions Shape Them. University of Chicago Press.

Dasgupta, S. (2014). On the Plurality of Grounds. Philosophers' Imprint 14.

Davidson, D. (1967). Causal relations. Journal of Philosophy, 64(21), 691-703.

Davis, P. J. (1981). Are There Coincidences in Mathematics? The American Mathematical Monthly, 88, 311-320.

deRosset, L. (2013). Grounding Explanations. Philosophers' Imprint 13.

Friedman, M. (1974). Explanation and scientific understanding. Journal of Philosophy, 71(1), 5-19.

Hart, H. L. A., \& Honoré, T. (1959). Causation in the Law. Oxford University Press.

Hicks, M. T., \& van Elswyk, P. (2015). Humean laws and scientific explanation. Philosophical Studies, 173, 433-443.

Horwich, P. (1982). Probability and Evidence. Cambridge University Press.

Kappes, Y., \& Schnieder, B. (2016). Anything at All - The Deepest and the Shallowest Question. Philosophisches Jahrbuch, 123(2), 543-565.

Kappes, Y. (forthcoming). Explanation by status as empty-base explanation. Synthese:1-21.

Kim, J. (1994). Explanatory knowledge and metaphysical dependence. Philosophical Issues, 5, 51-69.

Kitcher, P. (1981). Explanatory unification. Philosophy of Science, 48(4), 507-531.

Kitcher, P. (1989). Explanatory unification and the causal structure of the world. In K. Philip \& S. Wesley (Eds.), Scientific Explanation (pp. 410-505). University of Minnesota Press.

Kitcher, P. (2001). Science, truth, and democracy. Oxford University Press.

Lando, T. (2017). Coincidence and common cause. Noûs, 51(1), 132-151.

Lange, M. (2010). What Are Mathematical Coincidences (and Why Does It Matter)? Mind, 119(474), 307-340.

Lange, M. (2018). Transitivity, self-explanation, and the explanatory circularity argument against Humean accounts of natural law. Synthese, 195(3), 1337-1353.

Lewis, D. (1983). New work for a theory of universals. Australasian Journal of Philosophy, 61(4), 343-377.

Lewis, D. K. (1986). Causal explanation. In D. Lewis (Ed.), Philosophical Papers (Vol. II, pp. 214-240). Oxford University Press.

Marshall, D. G. (2015). Humean laws and explanation. Philosophical Studies, 172(12), 3145-3165.

Mellor, D. H. (1995). The facts of causation. Routledge.

Miller, E. (2015). Humean scientific explanation. Philosophical Studies, 172(5), 1311-1332.

Morreall, J. (1979). The Evidential Use of Because. Paper in Linguistics, 12(1-2), 231-238.

Nummela, E. (1987). No Coincidence. The Mathematical Gazette, 71(456), 147.

Owens, D. (1992). Causes and Coincidences. Cambridge University Press.

Roski, S. (2018). Grounding and the explanatory role of generalizations. Philosophical Studies, 175(8), 1985-2003.

Schaffer, J. (2017a). Laws for metaphysical grounding. Philosophical. Issues, 27(1), 302-321.

Schaffer, J., (2017b). The Ground Between the Gaps. Philosophers' Imprint 17.

Schnieder, B. (2010). A Puzzle About 'because.' Logique Et Analyse, 53(211), 317-343.

Schnieder, B. (2011). A logic for 'because'. Review of Symbolic Logic, 4(3), 445-465. 
Schnieder, B. (2015). The asymmetry of 'because.' Grazer Philosophische Studien, 91(1), 131-164. Skow, B. (2016). Reasons Why. Oxford University Press.

Sober, E. (1984). Common cause explanation. Philosophy of Science, 51(2), 212-241.

Publisher's Note Springer Nature remains neutral with regard to jurisdictional claims in published maps and institutional affiliations. 